\title{
RESULTADOS DO TRATAMENTO DO CARCINOMA ESPINOCELULAR ANAL E DO SEU PRECURSOR EM DOENTES HIV-POSITIVOS
}

\author{
Sidney Roberto Nadal*, Sergio Henrique Couto Horta, Edenilson eduardo Calore, Carmen Ruth Manzione \\ Trabalho foi realizado pelas Equipes de Proctologia e Patologia do Instituto de Infectologia Emilio Ribas, São Paulo, Brasil
}

\author{
*Correspondência \\ Rua Dr. Virgilio de Carvalho \\ Pinto, 381 apto. 23 \\ São Paulo/SP - Brasil \\ Cep: $05415-030$ \\ Tel./Fax (11) 3337-4282 \\ srnadal@terra.com.br
}

\begin{abstract}
RESUMO
OBjetivo. A incidência de carcinomas espinocelulares anais vem aumentando nos doentes HIV-positivos. O tratamento consiste de radio e/ou quimioterapia, eventualmente seguidos pela ressecção do tumor. $O$ objetivo é avaliar o seguimento desses doentes para verificar as recidivas e a evolução do NIAA para câncer. Apresentação da casuística da Equipe Técnica de Proctologia do Instituto de Infectologia Emílio Ribas, de São Paulo.

Métodos. Acompanhamos 45 doentes HIV-positivos portadores de carcinoma espinocelular anal e seu precursor no período de 1996 a 2006. Eram 30 neoplasias intra-epiteliais anais de alto grau (NIAA), tratadas com ressecção local e 15 carcinomas invasores do canal anal. Nove das 15 lesões invasivas foram submetidas ao esquema de Nigro isolado ou associado à ressecção local, amputação abdominoperineal ou colostomia para derivação.

REsultados. No seguimento ambulatorial das NIAA tivemos recidiva em 16,7\% e não observamos evolução para carcinoma, num período mínimo de três anos. Entre os nove tumores submetidos ao esquema de Nigro, com ou sem operação complementar, cinco estão livres de tumor. Nenhum tratamento foi feito em cinco doentes. Em três, devido às péssimas condições clínicas, e em dois, pois recusaram tratamento. Outro teve diagnóstico na vigência de oclusão intestinal sendo submetido à colostomia de derivação.

Conclusão. Concluímos que a NIAA pode reincidir após excisão local em doentes HIV-positivos, mas não evolui para carcinoma invasor e que os doentes de câncer anal invasor podem ser tratados da mesma maneira que a população soronegativa para o HIV desde que as condições clínicas o permitam.
\end{abstract}

Unitermos: Carcinoma de células escamosas. Radioterapia. Quimioterapia. Carcinoma intra-epitelial. Infecções por papillomavirus. Aids.

\section{INTRODUÇÃO}

A incidência do carcinoma anal corresponde a 1,5\% de todos os tumores do aparelho digestório, e entre $2 \%$ e $4 \%$ dos tumores colo-retais ${ }^{1-2}$. Acomete mais mulheres do que homens, na proporção de $5: 1^{3}$. Entretanto, sua freqüência é 25 a 50 vezes maior entre os portadores do vírus da imunodeficiência adquirida (HIV), demonstrando mudança na epidemiologia dessa afecção ${ }^{2,4}$. Esse aumento vem sendo notado desde os anos 80, particularmente entre os homossexuais masculinos ${ }^{5}$, e parece estar intimamente associado à infecção pelo Papilomavírus Humano (HPV) ${ }^{6-7}$. Além disso, vários autores acreditam que o esquema de drogas anti-retrovirais, atualmente usado pelos portadores do HIV, aumenta a expectativa de vida desses doentes. Desta forma, o estado de imunodepressão crônico e de maior duração pode predispor ao aparecimento de maior número de tumores malignos ${ }^{8-9}$.

$\mathrm{Na}$ literatura médica estão descritos vários fatores de risco para - carcinoma anal. São eles: a imunodepressão causada pela infecção pelo HIV; a associação com a infecção pelo HPV; a contagem sérica de linfócitos T CD4+ inferior a $100 / \mathrm{mm}^{3}$; a contagem viral elevada do HIV sugerindo ausência ou ineficácia do tratamento e denotando imunodepressão iminente; e a presença de displasia acentuada ${ }^{10-12}$, também conhecida como neoplasia intra-epitelial anal de alto grau (NIAA). Associadas a essas, outras situações de risco também foram observadas, tais como o trauma local e a inflamação crônica e prolongada, o sexo anal receptivo e o tabagismo².

A importância da imunodepressão e da infecção pelo HIV em relação a esse tumor se nota quando reparamos que a doença, antes mais freqüente nas mulheres acima dos 60 anos de idade 3,13 , tornouse mais comum nos homens entre 30 e 40 anos $^{14}$. Uma vez que o carcinoma anal é raro entre os soronegativos para o HIV e sua incidência vem crescendo entre os doentes de imunodeficiência adquirida (Aids), esse vírus poderia ser o co-fator que o HPV necessita para induzir às displasias que podem progredir para carcinoma ${ }^{4}$.

Muitos relatos têm indicado o tratamento não operatório com radio e quimioterapia que possui altos índices de cura e preserva o esfíncter anal|5,16. A expectativa de sobrevivência de $90 \%$ em cinco anos é referida com o uso de radioterapia em aplicações fracionadas, que somadas atingem entre 4500 cGy e 5500 cGy, associada à mitomicina $\mathrm{C}$ e ao 5-fluorouracil, endovenosos ${ }^{16-17}$. Quando não há resposta, o tratamento operatório com ressecção local ou amputação abdominoperineal estão indicados ${ }^{18}$. Para os 
NADAL SR ET AL.

doentes HIV-positivos o esquema terapêutico deve ser o mes$\mathrm{mo}^{15,19}$.

O prognóstico é pior entre os doentes imunocomprometidos. Esses apresentam mais metástases linfonodais, maiores taxas de recidiva, menor expectativa de vida ${ }^{10,20}$, e pior resposta à radio e à quimioterapia associadas ${ }^{12}$. Estudos comparando doentes imunodeprimidos e imunocompetentes vêm mostrando, respectivamente, envolvimento linfonodal em $60 \%$ e $17 \%$, recidivas em $75 \%$ e $6 \%$, boa resposta à radio e quimioterapia em $62 \%$ e $85 \%$, toxicidade a esse tratamento em $80 \%$ e $30 \%$, e sobrevivência global de I,4 e 5,3 anos 10,12,20. Todavia, naqueles que receberam o esquema de drogas anti-retrovirais ${ }^{21}$, o número de linfócitos $T$ CD4+ aumentou ${ }^{22}$ e o prognóstico melhorou"', sugerindo melhora da imunidade e controle da doença. Além disso, quantidade de displasias diminuiu apesar de haver discordância quanto a esse fato ${ }^{23}$

Poucos artigos sobre carcinoma anal em doentes HIV-positivos vêm sendo publicados. Desta maneira, decidimos avaliar o seguimento desses doentes para verificar as recidivas e a evolução do NIAA para câncer.

\section{Métodos}

Entre julho de 1996 e junho de 2006, atendemos 45 doentes HIV-positivos com câncer anal ou NIAA, seu provável precursor. A maioria era masculina $(97,7 \%)$ com idades variando entre 23 e 55, com média de 38,5 anos. Trinta apresentavam NIAA e 15 tinham carcinomas espinocelulares localizados no canal anal.

Os doentes com NIAA foram diagnosticados durante avaliação histológica das peças ressecadas de 23 condilomas anais, quatro doenças de Bowen, duas úlceras idiopáticas do canal anal e uma fistulectomia. Todos referiram tratamento anterior para condilomas anais. O tipo do HPV foi identificado com o método da reação em cadeia da polimerase (PCR) realizado no material obtido dos blocos de parafina dos espécimens ressecados.

Suspeitamos dos tumores malignos durante o exame físico do doente e o diagnóstico foi confirmado pela avaliação histológica. Todos os doentes foram atendidos entre 1996 e 2006, período em que o atual esquema anti-retroviral estava disponível. E todos se diziam aderentes ao tratamento da infecção pelo HIV. Realizamos o exame físico e indicamos as radiografias do tórax e a tomografia computadorizada do abdome e da pelve para conhecer - estádio da doença neoplásica, utilizando as classificações do American Joint Committee on Cancer/ Union Internationale Contre le Cancer (AJCC/UICC) (Tabela I)24. Nove doentes estavam no estádio II (20\%) e seis no estádio III (I3,4\%).

Para os doentes com NIAA, decidimos que a ressecção local, já realizada, foi suficiente como tratamento da doença anal. Para aqueles com carcinoma, a radio e quimioterapia combinada seria realizada inicialmente, desde que houvesse boas condições clínicas e que a operação de emergência não fosse necessária. Todos os doentes no estádio II foram submetidos a radio e quimioterapia. A radioterapia foi fracionada em doses que somadas totalizaram de 4.500 a 5.040 cGy e o esquema quimioterápico incluiu 5-fluoruacil e mitomicina $\mathrm{C}$, endovenosos. Nesse grupo, incluímos um doente com tumor de pequenas dimensões, submetido à biópsia excisional. O tumor comprometia microscopicamente as margens da área ressecada. Outro doente foi submetido à colostomia de alça para derivação do trânsito intestinal antes do esquema adjuvante. Entre os doentes no estádio III, dois recusaram qualquer tratamento, um foi submetido à colostomia em alça para derivação do trânsito intestinal devido obstrução tumoral e três não possuíam condições clínicas aceitáveis para a radio e quimioterapia, devido ao grau avançado da infecção pelo HIV.

Nos doentes com NIAA, o seguimento consistiu de avaliação semanal até cicatrização completa, retornos mensais até o sexto mês, passando a semestral, desde que não houvesse doença perianal. Realizamos biópsias das áreas suspeitas, e sempre que necessárias, com auxílio do colposcópio e ácido acético ${ }^{25}$.

Naqueles submetidos a radio e quimioterapia fizemos avaliações trimestrais e retiramos material para biópsia da área da fibrose, bem como das áreas suspeitas e das recidivas, 30 dias após o término do esquema terapêutico e depois a cada três meses.

\section{Resultados}

Todos os doentes com NIAA tinham infecção pelo HPV sugerida pelo exame histológico. O PCR identificou o tipo do HPV em 13 doentes. Eram sete $(53,8 \%)$ com os tipos não-oncogênicos 6 e II, quatro com os oncogênicos 18 e 31 (30,8\%), o tipo 53, de oncogenicidade desconhecida, em um $(7,7 \%)$, e em outro o tipo viral é desconhecido (7,7\%). Em três, o HPV não foi identificado. Todos permaneceram livres da doença e não houve progressão para carcinoma invasor num período que variou entre três e seis anos. No entanto, $17(56,7 \%)$ apresentaram recidiva do condiloma, sendo cinco deles com NIAA (16,7\%). A presença do HPV pôde ser observada em seis (40\%) dos doentes com carcinoma invasor. Essa infecção foi diagnosticada pelas características histológicas como coilocitose e inclusões virais intracelulares.

Quanto aos portadores do câncer anal, oito (88,8\%) daqueles no estádio II submetidos à radio e quimioterapia apresentaram resposta completa e permanecem livres da doença após seguimento de cinco a oito anos (média de sete anos). Dois relataram cefaléia e fraqueza durante dois ou três dias após a quimioterapia. $\mathrm{O}$ doente submetido à colostomia antes do tratamento teve a estomia fechada após seis meses. Submetemos aquele $(11,2 \%)$ que não respondeu ao tratamento à amputação abdominoperineal. Ele permanece vivo e livre da doença após cinco anos. Em quatro doentes, a radiação resultou em úlceras que não cicatrizaram, entretanto, as biópsias seriadas não revelaram doença neoplásica. A amputação abdominoperineal foi proposta para controle da úlcera, apesar de não haver malignidade, mas dois recusaram a operação e nos outros, o estádio de imunodeficiência contra-indicou o procedimento. Os seis doentes no estádio III não receberam tratamento específico para o carcinoma. A sobrevivência variou de 20 dias a seis meses (média de três meses). A morte em cinco deles $(83,3 \%)$ foi provocada pelas doenças associadas à Aids e no outro $(16,7 \%)$ pela progressão do câncer. 


\section{Tabela I - Estádio (AJCC/UICC) dos 45 doentes HIV-positivos com carcinoma espinocelular anal ou NIAA. Instituto de Infectologia Emilio Ribas, 2006}

\begin{tabular}{ll}
\hline estádio & $\frac{\mathbf{n}(\%)}{30(66,6 \%)}$ \\
\cline { 2 - 2 } I in situ) & zero \\
II & $9(20 \%)$ \\
N & $6(13,4 \%)$ \\
III & zero \\
\hline
\end{tabular}

$\mathrm{AJCC}=$ American Joint Committee on Cancer;

UICC = Union Internationale Contre le Cancer.

NIAA = neoplasias intraepithelial anal de alto grau

Nós também observamos que os seis doentes com pior evolução tinham as contagens de linfócitos T CD4+ muito baixas (média de $9 / \mathrm{mm}^{3}$, variando entre 4 e 21 ), enquanto que nos demais, com melhor evolução, as contagens oscilaram entre 25 e $332 / \mathrm{mm}^{3}$ (média de $217 / \mathrm{mm}^{3}$ ) à época do diagnóstico. O teste do $t$ de Student mostrou diferença significante. $(p=0.005)$

\section{Discussão}

Desde os anos 1980, o aumento dos casos de carcinoma anal, associados à infecção pelo HPV, vem sendo observado ${ }^{6,26}$. Caso o HPV seja o agente etiológico, esse tipo de câncer poderá ser considerado como doença sexualmente transmissível ${ }^{6,7}$. Na literatura, a incidência do HPV entre doentes com carcinoma anal varia entre $35 \%$ e $60 \%{ }^{6-7}$. Em nossos casos, o HPV esteve presente em 100\% das NIAAs e em 40\% dos carcinomas invasores. Todavia, - PCR só pôde ser realizado em 16 doentes, identificando os tipos virais em 13 deles $(81,2 \%)$. A literatura mostrou que a positividade da PCR para os tipos de HPV variou entre 32 e $93,8 \%$, quando tecidos fixados em formalina e parafinados foram avaliados ${ }^{27-30}$.

Os tipos 5, 8, 16, 18, 31 e 45 do HPV são os mais comuns na região anogenital e são considerados como oncogênicos. As oncoproteínas E6 desses tipos virais interagem com a E6AP ubiquitina ligase e se ligam a várias proteínas celulares, incluindo a p53, causando degradação celular ${ }^{31}$. Muitos autores acreditam que o potencial oncogênico desse vírus provavelmente venha dessa interaçãa $^{32-33}$. Em nosso estudo, observamos tanto os tipos oncogênicos como os não-oncogênicos do HPV provocando NIAA, sugerindo que esses últimos também podem estar associados às displasias mais acentuadas e ao risco de evolução para carcinoma invasor.

Embora o câncer anal seja mais freqüente em mulheres acima dos 60 anos, em nosso estudo a maioria era masculina (97,8\%) com média de 38,5 anos de idade. Essa discrepância foi devida ao perfil de nossos doentes: grande incidência de homens nas terceira e quinta décadas da vida e infecção pelo HIV, como descrito na literatura ${ }^{14}$. O aparecimento do carcinoma anal em idade mais jovem pode ocorrer devido à infecção pelo HPV, imunodepressão local e sistêmica provocada pela infecção pelo HIV e sexo anal receptivo ${ }^{7,20,22-23}$.

NIAA significa a presença de tumor restrito à mucosa. $O$ diagnóstico desses casos é mais raro e geralmente ao acaso ${ }^{34}$, embora haja especialistas em HIV fazendo rastreamento e seguimento com raspado para citologia (Papanicolaou) ${ }^{35-36}$. Nossos 30 doentes tiveram diagnóstico feito com estudo histológico dos espécimens retirados das doenças anais operadas. Algumas delas tiveram incidência aumentada entre os doentes soropositivos para o HIV, como por exemplo, o condiloma acuminado, a doença de Bowen (que é idêntica à NIAA), úlcera idiopática do canal anal e fístulal ${ }^{37}$. A ressecção local é o tratamento inicial preferencial para a

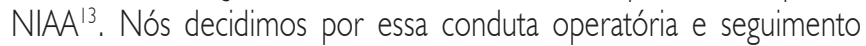
rigoroso. Nenhum dos nossos doentes evoluiu para câncer invasor, apesar de muitas dessas ocorrências estarem relatadas na literatura ${ }^{4}$. Identificamos cinco doentes (16,7\%) com recidiva da NIAA no seguimento, percentual inferior ao da literatura que registrou freqüências entre $64 \%$ e $73 \% 38-39$. Rotineiramente, tratamos todas as infecções induzidas pelo HPV com quatro aplicações tópicas, semanais, de podofilina a 25\% em vaselina sólida, quando abaixo da linha pectínea e ácito tricloroacético a 90\%, se acima dessa. As lesões remanescentes são submetidas à ressecção ou eletrocauterização. Notamos menos recidivas com esse esquema. Talvez, esse procedimento esteja evitando as recidivas da NIAA em nossos doentes. De qualquer maneira, todas os espécimens ressecados devem ser histologicamente examinados. Além disso, embora a ressecção local da NIAA possa parecer suficiente, o seguimento de forma oncológica é necessário para esses doentes.

O esquema com radio e quimioterapia combinadas é o tratamento inicial para os carcinomas do canal anal|5,16. Entretanto, nos doentes HIV-positivos, essa terapia pode ser contra-indicada conforme o estado de imunodepressão ou intensidade das infecções oportunistas, piorando o prognóstico ${ }^{40-41}$. Somado a isso, esses doentes têm menor tolerância ao tratamento que pode provocar complicações clínicas e alterações hematológicas, especialmente leucopenia ${ }^{16,41}$. Os efeitos tóxicos agudos e crônicos da quimioterapia podem ser exarcebados naqueles com contagens de linfócitos TCD4+ inferiores a $200 / \mathrm{mm}^{3}$, requerendo tratamento medicamentoso adicional $\left.\right|^{10,16,41}$. Não observamos qualquer efeito tóxico em nossos doentes, mas dois deles queixaram-se de cefaléia e fraqueza transitória e de curta duração após a quimioterapia. Ambos tinham contagens séricas de linfócitos T CD4+ inferior a $200 / \mathrm{mm}^{3}$.

Nós acompanhamos cinco doentes no estádio III, com Aids avançada, doenças oportunistas e leucopenia (contagens inferiores a $1.000 / \mathrm{mm}^{3}$ ), e que não possuíam condições clínicas para o tratamento específico para o câncer anal, e que morreram pelas complicações ocasionadas pela Aids. Dentre os nove doentes no estádio II que foram submetidos à radio e quimioterapia, oito tiveram resposta completa $(88,8 \%)$. Essa taxa de sucesso foi semelhante às obtidas para os doentes HIV-negativos com tumores no mesmo estádio, que variou de $70 \%$ a 92\%15,41.

Aqueles que apresentam resposta ausente ou parcial à radio e quimioterapia podem ser submetidos à nova quimioterapia, usando 5-fluorouracil e cisplatina associadas, e doses complementares de radioterapia com taxas de sucesso em até $50 \%$ dos $\operatorname{casos}^{42-43}$. O tratamento operatório é necessário para ressecar o tumor rema- 
NADAL SR ET AL.

nescente em caso de falência da terapia inicial, o que ocorre em 10\% a $15 \%$ das vezes, sendo a amputação abdominoperineal o procedimento mais realizado ${ }^{44-46}$. A radio e quimioterapia falhou em apenas um dos nossos doentes $(\mathrm{II}, 2 \%)$. Ele tinha menos que 200 linfócitos T CD4+, nenhuma doença oportunista e boas condições clínicas. Permanece sem doença residual, cinco anos após a amputação abdominoperineal.

Nós também observamos que os doentes com pior evolução tinham contagens muito baixas de linfócitos T CD4+ na época do diagnóstico do câncer anal. Resultados similares foram relatados por outros ${ }^{23}$, confirmando a importância do estado imunológico na gênese e na evolução desse tumor.

\section{Conclusão}

Baseados em nossos resultados, concluímos que a NIAA pode reincidir após excisão local em doentes HIV-positivos, mas não evolui para carcinoma invasor e que os doentes de câncer anal invasor podem ser tratados da mesma maneira que a população soronegativa para o HIV desde que as condições clínicas o permitam.

\section{Conflito de interesse: não há.}

\section{SUMMARY}

\section{OUTCOME OF TREATMENT OF ANAL SQUAMOUS CELL CARCINOMA AND ITS PRECURSOR IN HIV-INFECTED PATIENTS}

OBJECTIVE. Incidence of anal squamous cell carcinoma is increasing mainly among HIV-positive patients. Treatment consists of radiotherapy and chemotherapy, sometimes followed by tumor resection. The objective was to evaluate the follow-up of such patients to verify recurrences and evolution from HAIN to cancer. This is a report of cases treated at the "Instituto de Infectologia Emilio Ribas", Sao Paulo, Brazil.

Methods. We attended 45 HIV-positive patients between July 1996 and June 2006. Most were male (97.7\%), with ages ranging from 23 to 55 years (mean: 38.5 years). Thirty patients had high grade anal intra-epithelial neoplasia (HAIN), treated with local resection, and 15 with anal canal invasive squamous cell carcinoma were first submitted to chemo radiation, while biopsies were obtained during follow-up.

RESULTS. Patients with HAIN had recurrences in $16.7 \%$ of cases and remained cancer free for up to five years. Chemoradiation was not possible in five patients with invasive carcinoma (40\%) because three had advanced AIDS and two refused treatment. Eight (88.8\%) out of nine patients had complete response to chemoradiation and remained cancer free for a period from three to six years. Chemoradiation failed in the ninth patient: abdominal perineal resection was performed, and there was no recurrence over a fiveyear period.

CONCLUSION. We concluded that HAIN can recur after local resection in HIV-positive patients but does not evolve to invasive carcinoma. Invasive cancer can be treated in the same way as in HIV seronegative persons, when clinical conditions permit. [Rev Assoc Med Bras 2007; 53(4): 365-9]
KEY WORDS: Carcinoma, squamous cell. Radiotherapy. Drug Therapy. Carcinoma in situ. Papillomavirus infections. Aids.

\section{REFERÊNCIA}

I. Stearns MW Jr, Urmacher C, Sternberg SS, Woodruff J, Attiyeh F. Cancer of the anal canal. Curr Probl Cancer. 1980;4:1-44.

2. Ryan DP, Campton CC, Mayer RJ. Carcinoma of the anal canal. N Engl J Med. 2000;342(I I):798-800

3. Moscicki AB, Hills NK, Shiboski S, Darragh TM, Jay N, Powell K et al. Risk factors for abnormal anal cytology in young heterosexual women. Cancer Epidemiol Biomarkers Prev. 1999;8:173-8.

4. Modesto VL, Gottesman L. Sexually transmitted diseases and anal manifestations of AIDS. Surg Clin North Am. 1994;74:1433-64.

5. Lytwyn A, Salit IE, Raboud J, Chapman W, Darragh T, Winkler B, et al. Interobserver agreement in the interpretation of anal intraepithelial neoplasia. Cancer. 2005; 103:1447-56.

6. Frisch M, Fenger C, van den Brule AJ, Sorensen P, Meijer CJ, Walboomers JM, et al. Variants of squamous cell carcinoma of the anal canal and perianal skin and their relation to human papillomaviruses. Cancer Res. 1999;59:753-7.

7. Gervaz P, Allal AS, Villiger P, Buhler L, Morel P. Squamous cell carcinoma of the anus: another sexually transmitted disease. Swiss Med Wkly. 2003; 133:353-9.

8. Berretta M, Cinelli R, Martellotta F, Spina M, Vaccher E, Tirelli U. Therapeutic approaches to AIDS-related malignancies. Oncogene. 2003;22:6646-59

9. Berry JM, Palefsky JM, Welton ML. Anal cancer and its precursors in HIV-positive patients: perspectives and management. Surg Oncol Clin N Am. 2004; 13:355-73.

I 0. Kim JH, Sarani B, Orkin BA, Young HA, White J, Tannebaum I et al. HIV-positive patients with anal carcinoma have poorer treatment tolerance and outcome than HIV-negative patients. Dis Colon Rectum. 2001;44:1496-502.

I I. Place RJ, Huber PJ, Simmang CL. Anorectal lymphoma and AIDS: an outcome analysis. J Surg Oncol. 2000;73:1-4.

12. Sobhani I, Vuagnat A, Walker F, Vissuzaine C, Mirin B, Hervatin F et al. Prevalence of high-grade dysplasia and cancer in the anal canal in human papillomavirus-infected individuals. Gastroenterology. 200। ; 1 20:857-66

13. Klas JV, Rothenberger DA, Wong WD, Madoff RD. Malignant tumors of the canal anal: the spectrum of disease, treatment and outcomes. Cancer. 1999;85:1686-93.

I 4. Friedman HB, Saah AJ, Sherman ME, Busseniers AE, Blackwelder WC, Kaslow RA, et al. Human papillomavirus, anal squamous intraepithelial lesions, and human immunodeficiency virus in a cohort of gay men. J Infect Dis 1998; 178:45-52.

15. Cleator S, Fife K, Nelson M, Gazzard B, Phillips R, Bower M. Treatment of HIV-associated invasive anal cancer with combined chemoradiation. Eur J Cancer. 2000;36:754-8.

16. Ferrigno R, Nakamura RA, Dos Santos Novaes PE, Pellizzon AC, Maia MA, Fogarolli RC, et al. Radiochemotherapy in the conservative treatment of anal canal carcinoma: retrospective analysis of results and radiation dose effectiveness. Int J Radiat Oncol Biol Phys. 2005;61: |136-42.

17. Nigro ND, Vaitkevicius VK, Considine B. Combined therapy for cancer of the anal canal: a preliminary report. Dis Colon Rectum. 1974:17:354-6.

18. Valentini V, Mantello G, Luzi S, Macchia G, Manfrida S, Smaniotto D. Cancer of the anal canal and local control. Rays. 1998;23:586-94.

19. Lim ST, Levine AM. Non-AIDS-Defining Cancers and HIV Infection. Curr Infect Dis Rep. 2005;7:227-234.

20. Vatra B, Sobhani I, Aparicio T, Girard PM, Puy Montbrun TD, Housset $M$, et al. Caractéristiques cliniques, thérapeutiques et prognostiques des carcinomes épidemoides du canal anal chez les malades VIH positifs Gastroenterol Clin Biol. 2002;26:I50-6. 
2 I. Palefsky JM, Holly EA, Ralston ML, Da Costa M, Bonner H, Jay N, et al. Effect of highly active antiretroviral therapy on the natural history of anal squamous intraepithelial lesions and anal human papillomavirus infection. J Acquir Immune Defic Syndr. 2001;28:422-8.

22. Stadler RF, Gregorcyk SG, Euhus DM, Place RJ, Huber PJ, Simmang $\mathrm{CL}$. Outcome of HIV-infected patients with invasive squamous-cell carcinoma of the anal canal in the era of highly active antiretroviral therapy. Dis Colon Rectum. 2004;47:1305-9.

23. Clifford GM, Polesel J, Rickenbach M, Dal Maso L, Keiser O, Kofler A, et al. Cancer risk in the Swiss HIV Cohort Study: associations with immunodeficiency, smoking, and highly active antiretroviral therapy. J Natl Cancer Inst. 2005;97:425-32.

24. American Joint Committee on Cancer. Anal cancer. In Greene FL, Page DL, Fleming ID, et al., editors. AJCC cancer staging manual. New York: Springer-Verlag; 2001. p.139.

25. Nadal SR, Manzione CR. Infecção perianal recidivante pelo Papilomavirus humano. Rev Assoc Med Bras. 2001;47:278-9.

26. Landis SH, Murray T, Bolden S, Wingo PA. Cancer statistics, 1999. CA Cancer J Clin. 1999:49:8-31, I.

27. Adams V, Moll C, Schmid M, Rodrigues C, Moos R, Briner J. Detection and typing of human papillomavirus in biopsy and cytological specimens by polymerase chain reaction and restriction enzyme analysis: a method suitable for semiautomation. J Med Virol. 1996;48:161-70.

28. Dabic MM, Hlupic L, Babic D, Jukic S, Seiwerth S. Comparison of polymerase chain reaction and catalyzed signal amplification in situ hybridization methods for human papillomavirus detection in paraffinembedded cervical preneoplastic and neoplastic lesions. Arch Med Res. 2004;35:5। I-6

29. Varnai AD, Bollmann M, Griefingholt $H$, Speich N, Schmitt C, Bollmann $R$, et al. HPV in anal squamous cell carcinoma and anal intraepithelial neoplasia (AIN) Impact of HPV analysis of anal lesions on diagnosis and prognosis. Int J Colorectal Dis. 2006;21:135-42.

30. Venturoli S, Zerbini M, La Placa M Jr, D'Antuono A, Negosanti M, Gentilomi G, et al. Evaluation of immunoassays for the detection and typing of PCR amplified human papillomavirus DNA. J Clin Pathol. 1998;51:143-8

31. Zanier K, Charbonnier S, Baltzinger M, Nomine Y, Altschuh D, Trave G. Kinetic analysis of the interactions of human papillomavirus E6 oncoproteins with the ubiquitin ligase E6AP using surface plasmon resonance. J Mol Biol. 2005;349:40 I-12.

32. Crook T, Vousden KH. Properties of p53 mutations detected in primary and secondary cervical cancers suggest mechanisms of metastasis and involvement of environmental carcinogens. EMBO J. 1992; 1 1:393540.

33. Ho L, Chan SY, Chow V, Chong T, Tay SK, Villa LL, et al. Sequence variants of human papillomavirus type 16 in clinical samples permit verification and extension of epidemiological studies and construction of a phylogenetic tree. J Clin Microbiol. 1991;29:1765-72.

34. Webber J, Fromm D. Photodynamic therapy for carcinoma in situ of the anus. Arch Surg. 2004;139:259-61.
35. Arain S, Walts AE, Thomas P, Bose S. The Anal Pap Smear: Cytomorphology of squamous intraepithelial lesions. Cytojournal. 2005;2:4.

36. Bakotic WL, Willis D, Birdsong G, Tadros TS. Anal cytology in an HIVpositive population: a retrospective analysis. Acta Cytol. 2005;49: 163-8.

37. Nadal SR, Manzione CR, Galvão VM, Salim VR, Speranzini MB. Perianal diseases in HIV-positive patients compared with a soronegative population. Dis Colon Rectum. 1999;42:649-54.

38. Chang GJ, Berry JM, Jay N, Palefsky JM, Welton ML. Surgical treatment of high-grade anal squamous intraepithelial lesions: a prospective study. Dis Colon Rectum. 2002;45:453-8.

39. Goldstone SE, Kawalek AZ, Huyett JW. Infrared coagulator: a useful tool for treating anal squamous intraepithelial lesions. Dis Colon Rectum. 2005;48:1042-54.

40. Chauveinc L, Buthaud X, Falcou MC, Mosseri V, De la Rochefordiere A, Pierga JY, et al. Anal canal cancer treatment: practical limitations of routine prescription of concurrent chemotherapy and radiotherapy. $\mathrm{Br}$ J Cancer. 2003;89:2057-6I.

4I. Esiashvili N, Landry J, Matthews RH. Carcinoma of the anus: strategies in management. Oncologist. 2002;7:188-99.

42. Martenson JA, Lipsitz SR, Wagner H Jr, Kaplan EH, Otteman LA, Schuchter LM, et al. Initial results of a phase II trial of high dose radiation therapy, 5-fluorouracil, and cisplatin for patients with anal cancer (E4292): an Eastern Cooperative Oncology Group study. Int J Radiat Oncol Biol Phys. 1996;35:745-9

43. Minsky BD, Hoffman JP, Kelsen DP. Cancer of the anal region. In: De Vita Jr VT, Hellman S, Rosenberg SA, editors. Cancer: principles \& practice of oncology. $6^{\text {th }}$ ed. Philadelphia: Lippincott-Williams \& Wilkins; 2001. p. 1319-42.

44. Faynsod M, Vargas HI, Tolmos J, Udani VM, Dave S, Arnell T, et al. Patterns of recurrence in anal canal carcinoma. Arch Surg. 2000;135:1090-3; discussion 1094-5.

45. Nilsson PJ, Svensson C, Goldman S, Glimelius B. Salvage abdominoperineal resection in anal epidermoid cancer. Br J Surg. 2002;89:1425-9.

46. Pocard M, Tiret E, Nugent K, Dehni N, Parc R. Results of salvage abdominoperineal resection for anal canal cancer after radiotherapy. Dis Colon Rectum. 1998;41:1488-93. 